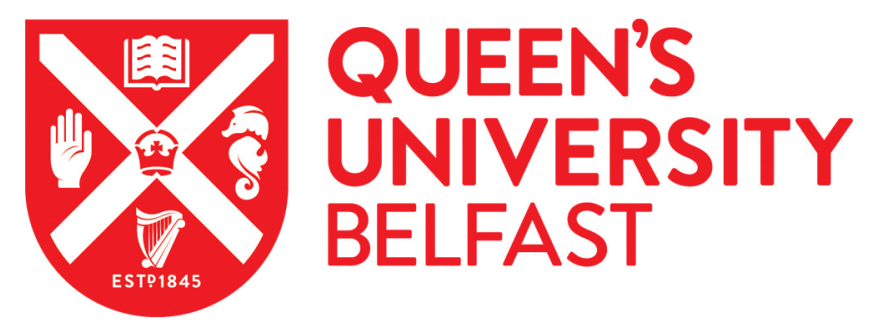

\title{
Immuno-Enriched Microspheres - Magnetic Blade Spray-Tandem Mass Spectrometry for Domoic Acid in Mussels
}

Geballa-Koukoula, A., Gerssen, A., Blokland, M. H., Elliott, C. T., Pawliszyn, J., \& Nielen, M. W. F. (2021). Immuno-Enriched Microspheres - Magnetic Blade Spray-Tandem Mass Spectrometry for Domoic Acid in Mussels. Analytical chemistry. https://doi.org/10.1021/acs.analchem.1c03816

Published in:

Analytical chemistry

Document Version:

Publisher's PDF, also known as Version of record

Queen's University Belfast - Research Portal:

Link to publication record in Queen's University Belfast Research Portal

Publisher rights

Copyright 2021 the authors. Published by American Chemical Society

This is an open access article published under a Creative Commons Attribution-NonCommercial-NoDerivs License

(https://creativecommons.org/licenses/by-nc-nd/4.0/), which permits distribution and reproduction for non-commercial purposes, provided the author and source are cited.

\section{General rights}

Copyright for the publications made accessible via the Queen's University Belfast Research Portal is retained by the author(s) and / or other copyright owners and it is a condition of accessing these publications that users recognise and abide by the legal requirements associated with these rights.

Take down policy

The Research Portal is Queen's institutional repository that provides access to Queen's research output. Every effort has been made to ensure that content in the Research Portal does not infringe any person's rights, or applicable UK laws. If you discover content in the Research Portal that you believe breaches copyright or violates any law, please contact openaccess@qub.ac.uk. 


\title{
Immuno-Enriched Microspheres - Magnetic Blade Spray-Tandem Mass Spectrometry for Domoic Acid in Mussels
}

\author{
Ariadni Geballa-Koukoula,* Arjen Gerssen, Marco H. Blokland, Christopher T. Elliott, Janusz Pawliszyn, \\ and Michel W.F. Nielen
}

Cite This: https://doi.org/10.1021/acs.analchem.1c03816

Read Online

ACCESS | 씨 Metrics \& More | 回 Article Recommendations

Supporting Information

\begin{abstract}
Paramagnetic microspheres can be used in planar array fluorescence immunoassays for single or multiplex screening of food contaminants. However, no confirmation of the molecular identity is obtained. Coated blade spray (CBS) is a direct ionization mass spectrometry (MS) technique, and when combined with triple quadrupole MS/MS, it allows for rapid confirmation of food contaminants. The lack of chromatography in CBS, though, compromises the specificity of the measurement for unequivocal identification of contaminants, based on the European Union (EU) regulation. Therefore, a rapid and easy-to-use immuno-magnetic blade spray (iMBS) method was developed in which immuno-enriched paramagnetic microspheres replace the coating of CBS. The iMBS-MS/MS method was fully optimized, validated in-house following the EU 2021/808 regulation, and benchmarked against a commercial lateral flow immunoassay (LFIA) for on-site screening of

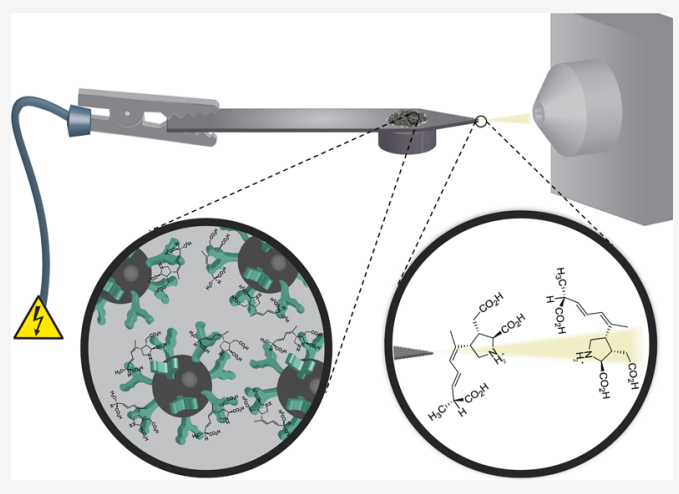
DA. The applicability of iMBS-MS/MS was further demonstrated by analyzing incurred mussel samples. The combination of immunorecognition and MS/MS detection in iMBS-MS/MS enhances the measurement's selectivity, which is demonstrated by the rapid differentiation between the marine toxin domoic acid (DA) and its structural analog kainic acid (KA), which cannot be achieved with the LFIA alone. Interestingly, this first-ever reported iMBS-MS/MS method is generic and can be adapted to include any other immuno-captured food contaminant, provided that monoclonal antibodies are available, thus offering a complementary confirmatory analysis approach to multiplex immunoassay screening methods. Moreover, thanks to its speed of analysis, iMBS-MS/ MS can bridge the logistics gap between future large-scale on-site testings using LFIAs and classical time-consuming confirmatory MS analysis performed in official control laboratories.
\end{abstract}

\section{INTRODUCTION}

Liquid or gas chromatography (LC- or GC-) tandem mass spectrometry (MS/MS) is regarded as the gold standard in terms of European regulation on confirmatory analysis of food contaminants. $^{1-3}$ However, LC- and GC-MS/MS analysis is time-consuming, which is a drawback for routine laboratories when large numbers of samples often need to be analyzed. ${ }^{4}$ Direct and ambient ionization mass spectrometry (AIMS) techniques can be used to shorten analysis time markedly. AIMS enables direct ionization of samples, with minimum or no sample pretreatment, and induces ionization under ambient conditions without chromatographic separation ${ }^{5}$ and many times directly from a surface. ${ }^{6}$ Just a few examples of such AIMS techniques are direct analysis in real time (DART), ${ }^{7}$ desorption electrospray ionization (DESI), ${ }^{8}$ and coated blade spray (CBS). ${ }^{9}$ DESI and DART were the first AIMS techniques developed ${ }^{7,8}$ and support ionization using a constant flow of carrier liquid and gas, respectively. Contrary, CBS employs a coated (on the tip) conductive metal strip, only requiring a small droplet of solvent for desorption and ionization, as in paper spray $^{10}$ and modified wooden-tip
ESI. ${ }^{11}$ The coating on CBS acts as a solid-phase microextraction (SPME) means to achieve selective enrichment of analytes from liquid samples or extracts. Following desorption of the compounds using a drop of organic solvent and high voltage application to the blade, spray ionization occurs without the requirement of additional gas or liquid flows. 9 The CBS's simplicity enhances the possibilities for future portable CBS-MS applications in food testing. ${ }^{12,13}$ CBS's most recent evolutionary aspect is magnetic blade spray (MBS), where paramagnetic surface-functionalized microparticles have replaced the coating for easy extraction and sample handling. ${ }^{14}$

Prior to confirmatory analysis by LC- or GC-MS/MS, rapid screening analysis is often performed. ${ }^{15}$ Screening methodologies include biorecognition-based sensors or assays with

Received: September 3, 2021

Accepted: October 25, 2021 
monoclonal antibodies (mAb), which can provide a quick qualitative or semiquantitative result for the presence of a targeted contaminant or a family of contaminants based on the cross-reactivity profile of the mAb employed. However, screening assays do not provide any structural information on the contaminant detected; thus, confirmatory analysis with LC- or GC-MS/MS is needed in the case of a non-compliant screening result. ${ }^{15,16}$ Apart from the well-known lateral flow immunoassay (LFIA), ${ }^{17,18}$ many other formats have been developed. Paramagnetic microspheres have been employed in fluorescent (flow)-based screening bioassays. Carboxyl groups on the surface allow for direct covalent coupling of $\mathrm{mAb}$ or proteins by EDC/NHS chemistry. ${ }^{19-21}$ Combining the features of biorecognition-based screening and direct MS/ MS may offer a novel and attractive rapid alternative workflow for confirmatory analysis. Only a few efforts have been made toward this direction of improved testing for contaminants, $^{22-26}$ underlining both its novelty and potential applicability. However, no demonstrations of direct immunocapturing and magnetic blade spray MS (iMBS) for rapid analysis have been published so far.

The present study showcases the iMBS approach, where mAbs have been covalently coupled to surface-functionalized paramagnetic microspheres for selective biorecognition and capturing of targeted analytes. Subsequent blade spray and triple quadrupole (QqQ)-MS/MS detection enable the ionization and unequivocal identification of the analytes without additional sample pretreatment. As a proof of concept, the method was developed to detect the marine shellfish toxin domoic acid (DA) and its structural analog, kainic acid (KA) in mussels. DA is an analog of the amino acids glutamate and proline. Specific phytoplankton species produce DA, which bioaccumulates in filter feeders such as shellfish, including scallops, oysters, and mussels. Consumption of DA-contaminated commodities may lead to amnesic shellfish poisoning (ASP); thus, it can cause severe central nervous system symptoms, such as disorientation, seizures, memory loss, and even death. ${ }^{27,28}$ The developed iMBS method to screen and confirm the presence of DA was validated according to the 2021 EU legislation $^{15}$ at three different target levels (TL) based on the maximum limit (ML) of $20 \mathrm{mg} / \mathrm{kg}^{29}$ over the course of three days and benchmarked against a commercial LFIA for on-site testing of DA.

\section{EXPERIMENTAL SECTION}

Chemicals and Materials. Acetonitrile and methanol of UHPLC-MS purity grade and ammonia solution (25\% v/v), formic acid (98\% v/v), acetic acid (98\% v/v), and DA and KA were purchased from Merck (Darmstadt, Germany). MilliQ water of $18.3 \mathrm{M} \Omega / \mathrm{cm}$ conductivity was obtained using a water purification system from Merck (Amsterdam, The Netherlands). Solutions of $5 \mathrm{mM}$ ammonium acetate and $5 \mathrm{mM}$ ammonium formate (Merck) were prepared in MilliQ water. Standard stock solutions of $1000 \mu \mathrm{g} / \mathrm{mL} \mathrm{DA}$, and $5000 \mu \mathrm{g} / \mathrm{mL}$ $\mathrm{KA}$, were prepared in acetonitrile/water $(10 / 90 \mathrm{v} / \mathrm{v})$.

For iMBS, polystyrene/divinylbenzene coated blades provided by Restek Corp. (Bellefonte, Pennsylvania, USA) were sonicated at $40{ }^{\circ} \mathrm{C}$ in methanol/formic acid $(50 / 50 \mathrm{v} / \mathrm{v})$ for $40 \mathrm{~min}$, to yield non-coated metal blades. The non-coated blades were used for the entire optimization of DA ionization with standard solutions. For adherence of paramagnetic microspheres, a type N48 neodymium magnetic disc $(3 \mathrm{~mm}$ $\times 2 \mathrm{~mm}$ ) from Supermagnete (Gottmadingen, Germany) was positioned under the tip of the non-coated blade. For the immuno-capturing part, MagPlex-C, paramagnetic carboxylated microspheres (MC10038, particle size of $5.6 \mu \mathrm{m}$ ), was purchased from Luminex Corp. (Austin, Texas, USA), and mouse mAbs against DA were provided by Queen's University Belfast. These antibodies previously demonstrated $24 \%$ crossreactivity with KA but no cross-reactivity toward any naturally co-occurring toxins. ${ }^{30}$ As described in the Luminex protocol, ${ }^{31}$ a carbodiimide covalent coupling procedure was followed for antibody immobilization on the paramagnetic microspheres. Briefly, $200 \mu \mathrm{L}$ of stock uncoupled paramagnetic microspheres was washed with MilliQ water and activated using monobasic sodium phosphate $\left(\mathrm{NaH}_{2} \mathrm{PO}_{4}\right)$ (Merck), sulfo-NHS (Nhydroxysulfosuccinimide), and EDC ( $N$-(3-dimethylaminopropyl)- $N$-ethylcarbodiimide hydrochloride) (Sigma-Aldrich, Zwijndrecht, The Netherlands). Next, mAbs for DA, $0.15 \mathrm{mg} /$ $\mathrm{mL}$ in MES (2-( $N$-morpholino)ethanesulfonic acid) (SigmaAldrich), were added to the activated paramagnetic microspheres for immobilization. After a $2 \mathrm{~h}$ incubation, the immuno-enriched paramagnetic microspheres were reconstituted in $200 \mu \mathrm{L}$ of PBS-TBN (phosphate-buffered saline with Tween-20, bovine serum albumin (BSA), and sodium azide) and stored in the refrigerator at $4{ }^{\circ} \mathrm{C}$ in a sealed dark Eppendorf tube. For the preparation of the PBS-TBN stock solution, a PBS solution containing $137 \mathrm{mM}$ sodium chloride $(\mathrm{NaCl}), 2.7 \mathrm{mM}$ potassium chloride $(\mathrm{KCl}), 10 \mathrm{mM}$ sodium hydrogen phosphate $\left(\mathrm{Na}_{2} \mathrm{HPO}_{4}\right)$, and $1.8 \mathrm{mM}$ potassium dihydrogen phosphate $\left(\mathrm{KH}_{2} \mathrm{PO}_{4}\right)$ (Merck, Darmstadt, Germany), having a $\mathrm{pH}$ of 7.4, was prepared in MilliQ water. The final storage buffer, PBS-TBN, was prepared by 10 -fold dilution of the PBS stock solution in MilliQ water containing $0.1 \% \mathrm{w} / \mathrm{v}$ BSA, $0.02 \% \mathrm{v} / \mathrm{v}$ Tween -20 , and $0.05 \% \mathrm{w} / \mathrm{v}$ sodium azide (Sigma-Aldrich).

For benchmarking, a commercially available LFIA screening test kit for DA, "Reveal 2.0 for ASP”, including LFIAs for DA and micro-perforated filter bags, was purchased from Neogen (Lansing, Michigan, USA). For the in-house validation, 21 blank mussel samples (Mytilus edulis), and for the applicability study, three naturally contaminated (incurred) samples, homogenized and stored at $-80{ }^{\circ} \mathrm{C}$, previously analyzed for the presence of DA by validated and accredited routine LCMS/MS and LC-UV methods, were provided in-house.

Instrumentation. The iMBS-MS/MS analysis was performed on a Micromass Quattro Ultima Pt QqQ-MS (Waters Corporation, Milford, MA, USA) equipped with a blade spray setup consisting of an $x-y-z$ stage and high-voltage plug from a Waters nanoESI ion source. A plastic clamp was used to secure the blade in place (Figure S1). Optimized operating conditions included positive ionization mode with $3.7 \mathrm{kV}$ spray voltage, $50 \mathrm{~V}$ cone voltage, $100{ }^{\circ} \mathrm{C}$ cone temperature, and $0.16 \mathrm{~mL} /$ min argon collision gas flow. Data were acquired in multiple reaction monitoring (MRM) mode, and two transitions were monitored; for DA, $m / z 312.1>266.1$ and $m / z 312.1>248.1$ at $12 \mathrm{eV}$ collision energy, and for KA, $m / z 214.1>168.1$ and $\mathrm{m} / z 214.1>122.1$ at 10 and $18 \mathrm{eV}$ collision energies, respectively. The data were acquired and processed using MassLynx software (Waters). From the chronograms acquired, area ion ratios of the two fragment ions for DA and KA were calculated and used for unequivocal confirmation of the identity of each substance according to the criteria. ${ }^{15}$

Methods. For method development and in-house validation, mussel samples were extracted using the previously developed and commercialized Neogen screening assay 
extraction protocol for efficient extraction of DA from samples. $^{32}$ In short, $1 \mathrm{~g}$ of the homogenized mussel sample was extracted using $30 \mathrm{~mL}$ of water. Vigorous manual agitation followed for $30 \mathrm{~s}$, , and afterward, a micro-perforated filter bag was used to extract further and remove the mussel residues. The final extract was stored in the refrigerator at $4{ }^{\circ} \mathrm{C}$ for further use.

To validate the iMBS-MS/MS method, portions of the blank mussel extracts were spiked at three different TLs, namely, 335 $\mathrm{ng} / \mathrm{mL}(0.5 \times \mathrm{TL}), 670 \mathrm{ng} / \mathrm{mL}(1 \times \mathrm{TL})$, and $1005 \mathrm{ng} / \mathrm{mL}$ $(1.5 \times \mathrm{TL})$, based on the theoretically calculated DA concentration in sample extracts from mussels contaminated at the ML of $20 \mathrm{mg} / \mathrm{kg}$. For the LFIA screening assay, $100 \mu \mathrm{L}$ of the extracts was further diluted with the buffer provided in a vial in the assay kit. Finally, $100 \mu \mathrm{L}$ of the mixture thus obtained was used to develop the LFIA.

The same spiked undiluted mussel extracts were also used for iMBS-MS/MS analysis. Ten microliters of the immunoenriched paramagnetic microspheres suspension was mixed in a 96-well plate with $100 \mu \mathrm{L}$ of the undiluted mussel extract and $100 \mu \mathrm{L}$ of MilliQ water. The mixture thus obtained was incubated at room temperature for $10 \mathrm{~min}$. Then, a magnetic plate was positioned underneath the 96-well plate to induce fast sedimentation and adherence of the immuno-enriched paramagnetic microspheres at the bottom of the 96-well plate. The supernatant was discarded, and the magnetically trapped microspheres were washed three times with $100 \mu \mathrm{L}$ of MilliQ water.

For the MS/MS analysis, the non-coated blade was placed at a $\pm 6 \mathrm{~mm}$ distance from the entrance cone of the ion source. While the spray voltage was at $0 \mathrm{kV}$, the incubated and washed paramagnetic microspheres were resuspended in $50 \mu \mathrm{L}$ of MilliQ water and retrieved from the 96-well plate to be pipetted onto the non-coated metal blade at the tip of the blade with the magnet underneath. Next, after $30 \mathrm{~s}$ of adherence time of the paramagnetic microspheres, the excess supernatant liquid was removed with a clean tissue. Then, $4 \mu \mathrm{L}$ of methanol/formic acid $(2.5 \% \mathrm{v} / \mathrm{v})$ was pipetted on top of the immuno-enriched paramagnetic microspheres to dissociate the antigen from the immobilized antibodies. After $1 \mathrm{~min}$, the binding of the $\mathrm{DA}$ from the $\mathrm{mAb}$ on the immuno-enriched paramagnetic microspheres has been disrupted, and a second 4 $\mu \mathrm{L}$ aliquot was pipetted because of evaporation of the first 4 $\mu \mathrm{L}$. Finally, the optimized spray voltage of $3.7 \mathrm{kV}$ was applied to the non-coated blade to obtain an ESI-like spray, and the ions formed were analyzed by MS/MS (Figure 1).

\section{RESULTS AND DISCUSSION}

Method Development. Optimization of Blade Spray and MS Conditions. Initial optimization was performed using DA solutions and non-coated blades aiming at optimum ionization and MS operating conditions. Ionization was performed at a distance of $\pm 6 \mathrm{~mm}$ between the tip of the blade and the cone inlet of the MS system, to prevent any loss of paramagnetic microspheres by vacuum suction at the final stage of the experimental design. The optimum spray/ desorption solution among the solutions tested was that of methanol/formic acid $(2.5 \% \mathrm{v} / \mathrm{v})$. For more information on the optimization of the spray/desorption solution, see the Supporting Information and Figure S2.

Furthermore, the matrix interferences were briefly investigated using a methanol/formic acid solution fortified with 0 , 0.1 , and $1 \% \mathrm{v} / \mathrm{v}$ blank mussel extracts, to mimic an estimate of

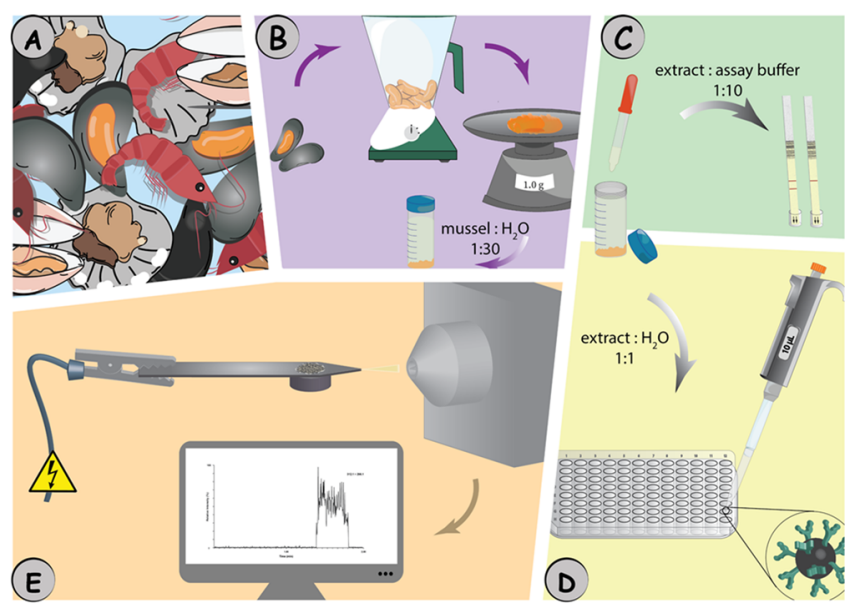

Figure 1. Workflow application of iMBS-MS/MS for analysis of contaminated shellfish samples. (A) Shellfish samples are collected on-site. (B) The selected shellfish commodity, i.e., mussels, is homogenized, weighed, and $1 \mathrm{~g}$ is extracted with $30 \mathrm{~mL}$ of distilled water. (C) The sample extract is further diluted with assay buffer for rapid on-site testing using a commercial screening LFIA, leading to a negative result (two lines, both test and control lines present) for any quantitative result lower than the $\mathrm{ML}$ of $20 \mathrm{mg} / \mathrm{kg}$. In contrast, it provides a positive result (control line only) for any readout of equal or more than $20 \mathrm{mg} / \mathrm{kg}$. (D) For a positive or ambiguous result, the same sample extract from step (B) can be used for confirmation using the developed iMBS; the extract is diluted to $1: 1$ with MilliQ water, and $200 \mu \mathrm{L}$ of the diluted extract is incubated with $10 \mu \mathrm{L}$ of immunoenriched paramagnetic microspheres. (E) After incubation, the final step is the deposition and fixation of the immuno-enriched paramagnetic microspheres on the blade's tip using a super magnet followed by dissociation with methanol/formic acid $(2.5 \% \mathrm{v} / \mathrm{v})$ and MS/MS confirmatory analysis.

the matrix residue after three washing cycles of the paramagnetic microspheres at the final experimental iMBS setup. The presence of mussel matrix residues did not alter the resulting chronograms for any of the solutions tested.

Finally, the spray voltage, cone voltage, and collision energy were optimized. Optimum conditions included $3.7 \mathrm{kV}$ spray voltage, $50 \mathrm{~V}$ cone voltage, and $12 \mathrm{eV}$ collision energy for the MRM transitions $m / z 312.1>266.1$ and $m / z 312.1>248.1$ of $\mathrm{DA}$ and 10 and $18 \mathrm{eV}$ for the MRM transitions $\mathrm{m} / z 214.1>$ 168.1 and $m / z 214.1>122.1$ of KA, respectively. For more information on the optimization of the MS operating conditions, see the Supporting Information.

When using the mussel extraction protocol (Figure 1B), the calculated concentration of DA in the extract of a contaminated sample at the TL is approximately $670 \mathrm{ng} / \mathrm{mL}$. Following a 1:1 dilution (Figure 1D), $33 \mathrm{ng}$ of DA will be theoretically available for incubation with the immunoenriched paramagnetic microspheres, but only a small fraction of this is expected to bind due to the limited antibody capacity available (Supporting Information). Therefore, the LOD/LOQ in direct MS/MS was assessed over a small dynamic range of 0-12 ng/mL only, using non-coated blades and DA spiked in methanol/formic acid $(2.5 \% \mathrm{v} / \mathrm{v})$ with a $0.1 \% \mathrm{v} / \mathrm{v}$ blank mussel matrix. The limit of quantitation (LOQ) was determined at $2 \mathrm{ng} / \mathrm{mL}$ and the limit of detection (LOD) at $0.6 \mathrm{ng} / \mathrm{mL}$. Since no chromatography is applied, it could be argued that the method's selectivity is compromised in any direct spray-MS/MS method. According to the latest version of the EU legislation $2021 / 808,{ }^{15}$ for substances with an 
established ML, four identification points (IPs) are required for the unequivocal identification of contaminants, one of which is obtained from the chromatographic separation and three from the MS/MS detection when two ion transitions are being monitored. It is stated in the EU 2021/808 document that all MS analyses shall be combined with a separation technique "that shows sufficient separation power and selectivity for the specific application". It may be argued that the high selectivity of immuno-capturing is by far superior versus a generic LC gradient separation using a C18 column, and therefore one IP point could be claimed for iMBS. In combination with the three IPs of MS/MS analysis, unequivocal identification of DA could be obtained using iMBS-MS/MS. To a further extent, according to Berendsen et al., ${ }^{33}$ the selectivity of a direct MS/MS method is assessed by the probability of interference, i.e., $P(I)$ value, which demonstrates the probability of the occurrence of other than the selected compound showing the same MS/MS characteristics. Having chosen the most selective MRM transitions for DA, $P(I)$ is assessed at $4.8 \times 10^{-5}$, which is still higher than the cutoff $P(I)$ value of $2 \times 10^{-7}$ for achieving a selective direct MS/MS method. Therefore, it is crucial that a direct MS/MS method features additional selectivity as provided by iMBS discussed in this paper. The immuno-capturing in iMBS adds selectivity to the overall MS/MS method, considering the specificity of the mAb that targets only DA and structural analogs; the latter can be differentiated in MS/MS based on their $m / z$.

iMBS-MS/MS of Marine Toxins in Shellfish. As a starting point to capture the $\mathrm{mAb}$-coupled paramagnetic microspheres, prototype magnetic blades consisting of a magnetic material with a copper strip for $\mathrm{HV}$ application were studied. These magnetic blades were provided by the Pawliszyn group and were previously used in the experimental setup described by Rickert et al. ${ }^{14}$ It was noticed that for the same DA solutions tested in the range of $0-12 \mathrm{ng} / \mathrm{mL}$, the signal was lower as compared to the non-coated metal blades used in our blade spray optimization experiments (cf. above), and due to the lower signal, the ion ratios were less robust using these prototype magnetic blades compared to non-coated metal blades (Figure S3). The apparent differences between the prototype magnetic blades and the non-coated metal blades were the conductivity of the material and the tip angle. With the non-coated blade, the entire surface is conductive until the tip end. However, the magnetic prototype material is nonconductive, and to compensate for this lack of conductivity, a copper strip is connected to the blade material. However, the copper strip does not cover the entire surface and, instead, ends a few millimeters away from the tip, resulting in reduced conductivity. In addition, the prototype magnetic blades' material is not as resistant to deformation as the non-coated blades, causing the tip to be less well defined and sharp. The tip is where the voltage is concentrated at the vertex of the blade, according to the first description of CBS by Gómez-Rios and Pawliszyn. ${ }^{9}$ Changes in the shape of the tip could result in alterations of the spray angle and the accumulation of the voltage, thus applying the same voltage setting in both blades will yield differences in the effective voltage and electric field at the tip. Consequently, we only used non-coated metal blades obtained by the removal of the coating from commercial CBS. Immuno-enriched paramagnetic microspheres were trapped onto these metal blades using a magnetic disc positioned under the tip. The trapping of the microspheres on the blade was characterized by SEM, where differences in the blade surface can be clearly seen between blank (Figure 2A) and paramagnetic microsphere-enriched surface (Figure 2B).

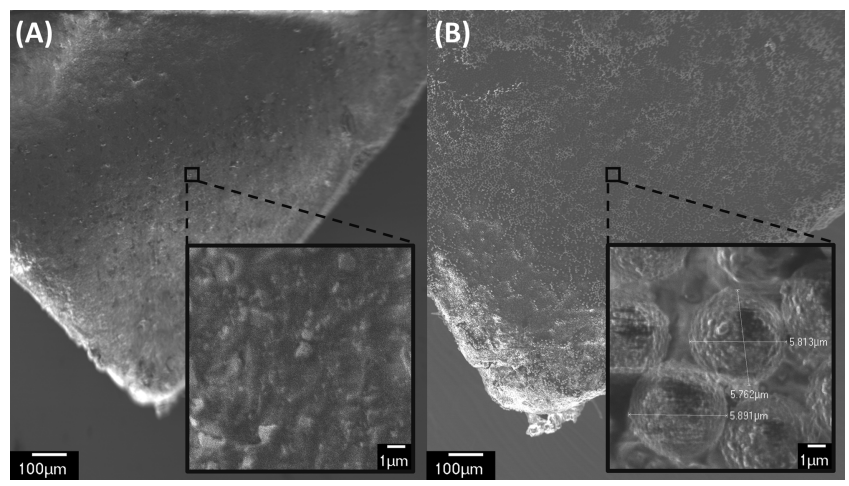

Figure 2. SEM images of (A) non-coated magnetic blades and (B) non-coated magnetic blades with captured paramagnetic microspheres.

In order to develop the final iMBS-MS/MS method, the biorecognition part was optimized with respect to the incubation time needed for binding between the immunoenriched paramagnetic microspheres and the DA in spiked sample extracts. More specifically, $10 \mu \mathrm{L}$ of immuno-enriched paramagnetic microspheres was incubated with $200 \mu \mathrm{L}$ of a $1: 1$ water dilution of the mussel extract spiked with DA at a level of $670 \mathrm{ng} / \mathrm{mL}$. The tested incubation times were $3,5,10$, and 20 min. No signal was observed for the MRM transitions of DA when incubating for $3 \mathrm{~min}$, indicating no binding between the $\mathrm{mAb}$ and $\mathrm{DA}$. The $5 \mathrm{~min}$ incubation produced half the area intensities in the reconstructed MRM chronograms as compared to the $10 \mathrm{~min}$ incubation. The latter turned out to be sufficient for quantitative analysis of lower concentrations. No significant changes were observed in the area intensities of the DA MRM transitions for the 10 and 20 min incubations, possibly due to saturation of the immobilized mAbs; therefore, a $10 \mathrm{~min}$ incubation was invariably used in the optimized protocol (Figure 3A). Finally, the amount of immuno-enriched paramagnetic microspheres to be used in each incubation was varied. Incubation for $10 \mathrm{~min}$ with $200 \mu \mathrm{L}$ of $1: 1$ diluted spiked $(670 \mathrm{ng} / \mathrm{mL})$ mussel extract and 5,10 , or $15 \mu \mathrm{L}$ of paramagnetic microspheres was tested. Five microliters of paramagnetic microspheres produced lower area intensities of the DA MRM transitions than the other volumes tested. Between 10 and $15 \mu \mathrm{L}$, similar results were obtained on the area intensities of the DA MRM transitions. However, with the use of $10 \mu \mathrm{L}$ of immuno-enriched paramagnetic microspheres, one $4 \mu \mathrm{L}$ drop of dissociation/spray solution onto the blade was sufficient for quantitative dissociation of DA from the immuno-enriched paramagnetic microspheres, and repetitive desorptions did not yield additional signals showing the correct ion ratios. In contrast, with $15 \mu \mathrm{L}$ of immuno-enriched paramagnetic microspheres, multiple desorption steps are needed for quantitative dissociation. Therefore, $10 \mu \mathrm{L}$ was found to be the most cost effective and appropriate volume to be used (Figure 3B).

To illustrate the feasibility of the iMBS-MS/MS approach, a blank mussel extract was incubated with immuno-enriched paramagnetic microspheres. The same extract was spiked with $\mathrm{DA}$ at $670 \mathrm{ng} / \mathrm{mL}$, and next $200 \mu \mathrm{L}$ of both the blank and fortified 1:1 diluted sample extract were incubated with $10 \mu \mathrm{L}$ 

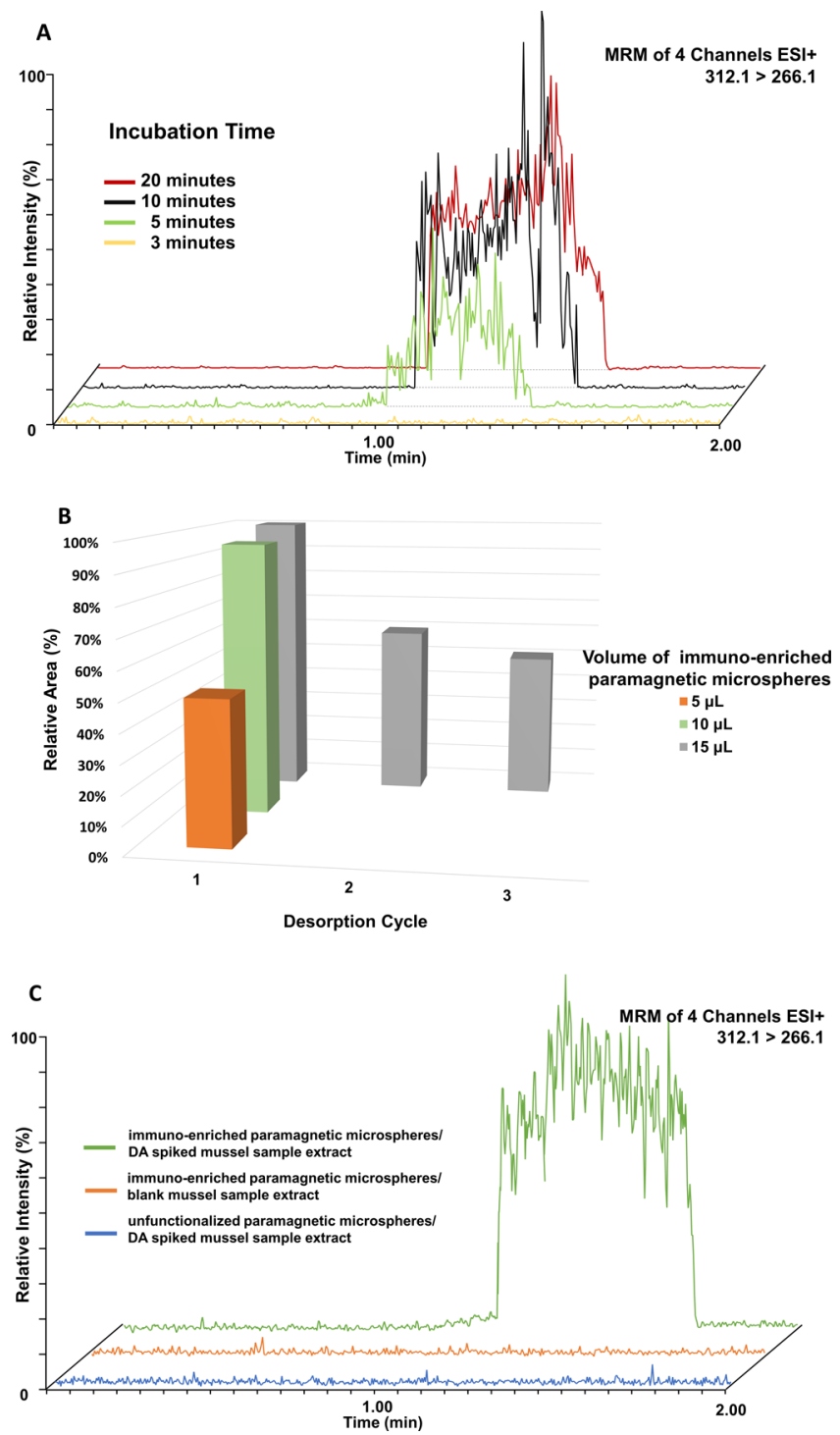

Figure 3. (A) Overlay chronograms of the $m / z 312.1>266.1$ transition obtained following iMBS-MS/MS, varying in incubation time. (B) Bar graphs of the relative area for the MRM transition $m / z$ $312.1>266.1$ versus the number of desorption cycles following iMBSMS/MS, varying in the volume of immuno-enriched paramagnetic microspheres. (C) Overlay chronograms of the $\mathrm{m} / z 312.1>266.1$ transition obtained following iMBS-MS/MS using immuno-enriched and unfunctionalized paramagnetic microspheres. For the exact conditions, see text.

of unfunctionalized paramagnetic microspheres and $10 \mu \mathrm{L}$ of immuno-enriched paramagnetic microspheres. The results, as expected, showed no signal for the blank sample matrix nor for the unfunctionalized paramagnetic microspheres. In contrast, DA MRM transition signals with the correct ion ratio of 0.29 (i.e., within the $\pm 40 \%$ relative deviation from the 0.34 ion ratio for $\mathrm{DA}^{15}$ ) were achieved for the immuno-enriched paramagnetic microspheres incubated with the spiked matrix sample (Figure 3C).

Opposed to the original CBS concept, in iMBS, the sorbent is not permanently bound to the blade but initially suspended in the sample extract. We could argue an arrangement where the immunosorbent is covalently coupled to the blade for simplicity of operation, but the extraction rate in such arrangement might be slower because of mass transfer limitations. Moreover, the option to optimize and/or apply the same immuno-enriched microspheres in a conventional planar array fluorescence immunoassay instrument would be sacrificed in that case.

Initial in-House Method Validations. To validate the developed iMBS-MS/MS approach and the rapid screening LFIA following the recent EU 2021/808 legislation, 21 blank mussel samples were provided in-house, extracted with the LFIA protocol, and the extracts were spiked at three different levels $(0.5 \times \mathrm{TL}, 1 \times \mathrm{TL}$, and $1.5 \times \mathrm{TL})$ and blank. Over the course of three days, seven samples were analyzed each day, and the results of the statistical analysis were used to assess the performance of the methods. Details on how the calculations were performed for the assessment of the validation criteria can be found in the Supporting Information.

Validation of the Screening LFIA Used for Benchmarking. To validate the commercial screening LFIA, the assessment levels were blank, $0.5 \times \mathrm{TL}$, and $1 \times \mathrm{TL}$, because of the inability to differentiate between 1 and $1.5 \times \mathrm{TL}$ (Figure 4A). For a semi-quantitative readout of the LFIAs, a custom $3 \mathrm{D}$ printed holder and smartphone readout were used to collect photographs (Figure S4) and measure the intensity of the test ( $\mathrm{T}$ ) and the control (C) lines and calculate the $\mathrm{T} / \mathrm{C}$ ratio (Supporting Information).

Regarding the specificity/sensitivity of the screening LFIA, sufficient discrimination was demonstrated between blank and spiked samples from the $0.5 \times$ TL level onward (Figure 4A and Table S1). Furthermore, based on the (semi-) quantitative smartphone reader, the intra-day repeatability results were $13.7,10.3$, and $13.9 \%$ at $0.5 \times \mathrm{TL}$ and $15.8,9.4$, and $16.8 \%$ at the $1 \times$ TL levels. The inter-day repeatability results were 14.0 and $14.6 \%$ at $0.5 \times$ and $1 \times \mathrm{TL}$, respectively. Moreover, the within-laboratory reproducibility results were 13.9 and $15.8 \%$ at $0.5 \times \mathrm{TL}$ and $1 \times \mathrm{TL}$, respectively. The acceptable $\% \mathrm{RSD}$ value for substances with an $\mathrm{ML}$ of $>1000 \mu \mathrm{g} / \mathrm{kg}$ is $<16 \%$, ${ }^{15}$ which means that only one value on the third day of $1 \times \mathrm{TL}$ measurements did not comply for a quantitative screening method. Consequently, for the time being, our smartphonebased LFIA for DA should be considered as a semi-quantitative screening method. Moreover, the calculated trueness values were 91 and $112 \%$ at the $0.5 \times$ and $1 \times \mathrm{TL}$, respectively, and within the acceptance range of 80 to $120 \%$ as stated in the legislation. ${ }^{15}$ Finally, the CC $\beta$ was calculated at $12.8 \mathrm{mg} / \mathrm{kg}$ (0.69 ratio $\mathrm{T} / \mathrm{C}$ )

Validation of the Newly Developed iMBS-MS/MS Method. The use of specific DA antibodies that isolate the analyte of interest, monitoring two MRM transitions, and assessing their ion ratio, assure the iMBS-MS/MS specificity. From the analysis of 21 blank mussel samples, no DA signal was observed, underlining the specificity of the iMBS-MS/MS approach. A clear signal was observed from the $0.5 \times \mathrm{TL}$ spiking level onward (Figure 4B,C). Moreover, the selected MRM transitions monitored and the respective ion ratios (Table S1) enable the confirmation of the identity of DA, without the need of an internal standard (I.S.) (Figure S4). The mean ion ratio for the MRM for DA was $0.34 \pm 0.14(\mathrm{~m} / z$ $248.1 / 266.1$ ) for all the 21 spiked samples analyzed. This ratio is identical to the ion ratio measured for DA in standard methanol/formic acid/mussel solutions. As a result, the ion ratio tolerance limit of the spiked mussel samples analyzed complies with the regulatory requirement of $\pm 40 \%$ relative deviation allowed by the EU $2021 / 808$ legislation. ${ }^{15}$ Thus, the method is demonstrated to be sufficiently specific/sensitive. 
A

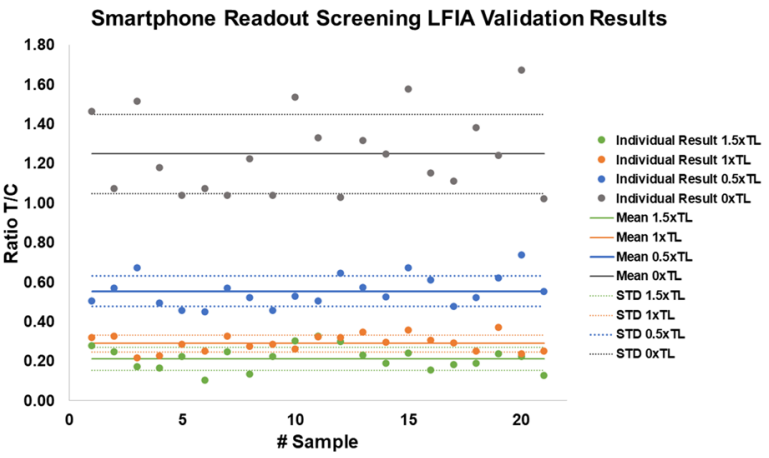

B
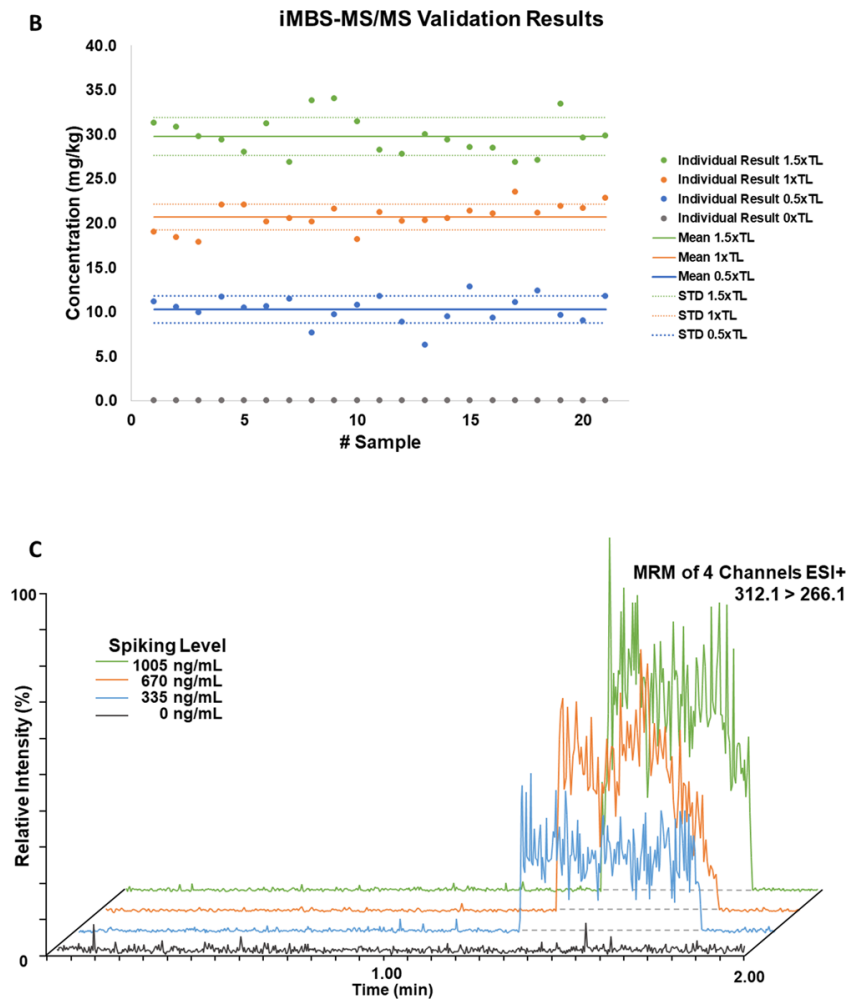

Figure 4. (A) Data analysis of results from 21 blank mussel samples and spiked versions thereof in screening LFIA. On the vertical axis is the intensity of the isolated blue channel of the test line over the control line. (B) Data analysis of results from 21 blank mussel samples and spiked versions thereof in iMBS-MS/MS. (C) iMBS-MS/MS chronograms of the $\mathrm{m} / z 312.1>266.1$ transition from paramagnetic microspheres/DA spiked sample at different target levels.

The intra-day repeatability results were $5.4,15.9$, and $13.0 \%$ at $0.5 \times \mathrm{TL}, 7.7,5.0$, and $3.9 \%$ at $1 \times \mathrm{TL}$, and 5.3, 7.6, and $7.0 \%$ at the $1.5 \times$ TL level, for days 1,2 , and 3, respectively. The inter-day repeatability results were $13.7,7.0$, and $7.1 \%$ for the $0.5 \times, 1 \times$, and $1.5 \times \mathrm{TL}$, respectively. Furthermore, the withinlaboratory reproducibility results were $13.9,6.1$, and $7.3 \%$ at $0.5 \times, 1 \times$, and $1.5 \times \mathrm{TL}$, respectively. All RSD\% values for the validation parameters assessed were lower than $16 \%$ and within the acceptance range, ${ }^{15}$ underlining the quantitative performance of the developed method in this range and a favorable comparison versus the LFIA screening assay. Moreover, the trueness values were calculated at 103,104 , and $99 \%$ for the $0.5 \times, 1 \times$, and $1.5 \times \mathrm{TL}$, respectively, which is within the acceptance range of 80 to $120 \% .{ }^{15}$ Finally, the CC $\alpha$ was 23.3 $\mathrm{mg} / \mathrm{kg}$, demonstrating that all samples of the $1.5 \times \mathrm{TL}$ and above were non-compliant. Therefore, the developed iMBSMS/MS method has been successfully validated as a quantitative confirmatory method over a limited range around the relevant ML level.

The specificity and applicability of the method was further demonstrated by the analysis of KA-spiked and DA-incurred mussel samples. KA is a structural analog of DA but has lower toxicity, and hence the EU does not regulate it. However, KA might interfere with the immuno-capturing in LFIA and iMBS due to its structural similarity. Indeed, using a blank mussel extract spiked with $\mathrm{KA}$ at $1005 \mathrm{ng} / \mathrm{mL}$ (corresponding to the $1.5 \times$ TL DA level), the LFIA screening test for DA yielded a false-positive result because of the inability of the used $\mathrm{mAb}$ to differentiate between structural analogs. However, when using iMBS-MS/MS, it becomes clear that the positive LFIA test result is caused by the presence of KA only since the characteristic ion transitions and respective area ion ratio of 0.34 belonging to DA were absent in the MS/MS data. In contrast, the characteristic product ions belonging to KA were detected with their corresponding area ion ratio of $0.21(\mathrm{~m} / z$ 122.1/168.1) (Figure 5B and Table 1).

Finally, following the workflow described in Figure 1, three DA-incurred mussel samples originating from the Netherlands
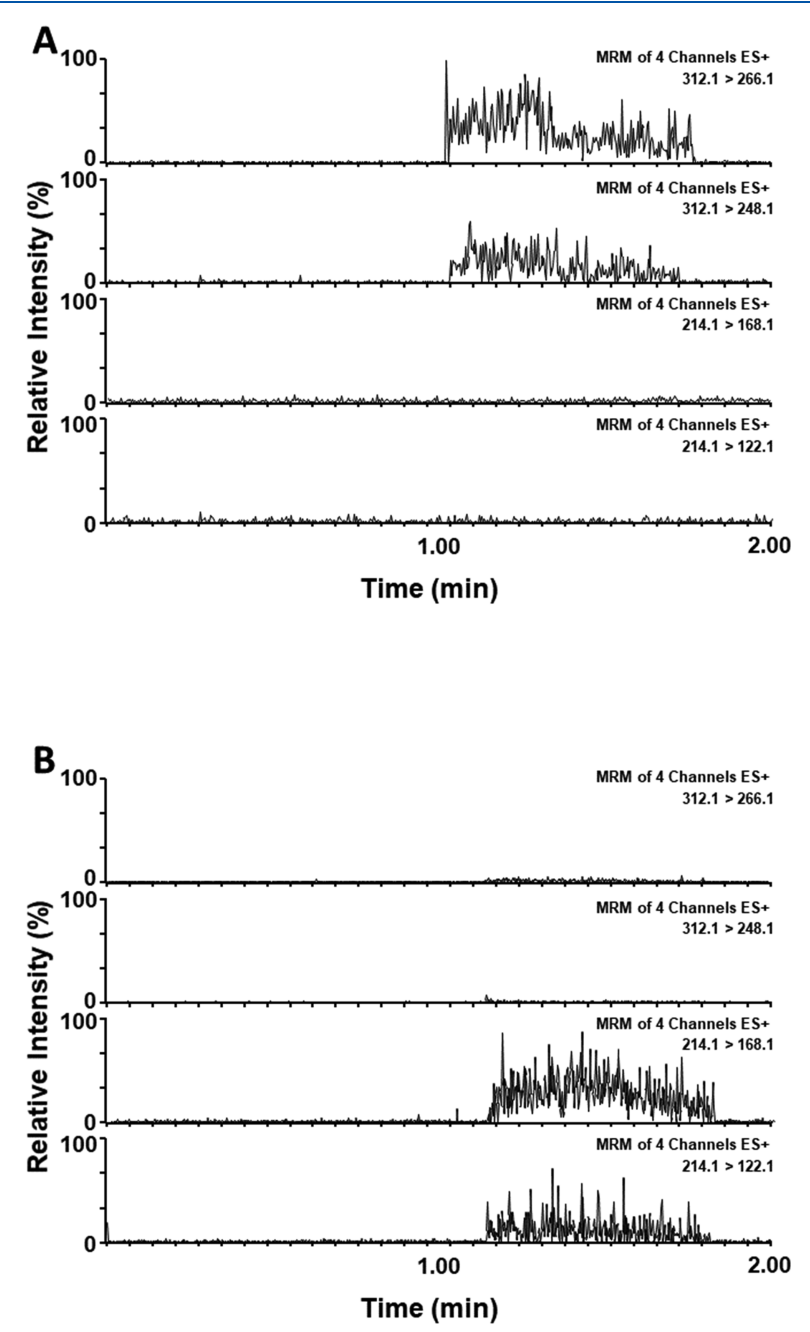

Figure 5. Examples of iMBS-MS/MS chronograms of (A) DA-spiked mussel extract and (B) KA-spiked mussel extract. For conditions, see text. 
Table 1. Results from iMBS-MS/MS Analysis and LFIA Screening of KA-Spiked Mussel Sample and DA-Incurred Mussel Samples ${ }^{a}$

\begin{tabular}{|c|c|c|c|c|c|c|}
\hline \multirow[b]{3}{*}{ sample } & \multicolumn{4}{|c|}{ iMBS-MS/MS } & \multirow[b]{3}{*}{$\begin{array}{l}\text { LFIA screening } \\
\text { result }(\mathrm{T} / \mathrm{C})\end{array}$} & \multirow[b]{3}{*}{$\begin{array}{l}\text { DA concentration based on the reference LC } \\
\text { MS/MS method }(\mathrm{mg} / \mathrm{kg})\end{array}$} \\
\hline & \multicolumn{2}{|r|}{ DA } & \multicolumn{2}{|r|}{$\mathrm{KA}$} & & \\
\hline & $\begin{array}{l}\text { ion } \\
\text { ratio }\end{array}$ & $\begin{array}{l}\text { calculated } \\
\text { concentration } \\
(\mathrm{mg} / \mathrm{kg})\end{array}$ & $\begin{array}{l}\text { ion } \\
\text { ratio }\end{array}$ & $\begin{array}{c}\text { calculated } \\
\text { concentration } \\
(\mathrm{mg} / \mathrm{kg})\end{array}$ & & \\
\hline KA spiked mussel extract & & & 0.21 & $27.5^{b}$ & 0.21 & \\
\hline incurred mussel \#1 & 0.37 & 23.5 & & & 0.23 & 20.0 \\
\hline incurred mussel \#2 & 0.36 & 31.5 & & & 0.17 & 39.0 \\
\hline incurred mussel \#3 & 0.39 & 35.6 & & & 0.09 & 46.6 \\
\hline
\end{tabular}

${ }^{a}$ The screening result is corrected versus the blank and then the intensity of the test line is divided by the control line (Supporting Information); for the exact conditions, see text. ${ }^{b}$ The calculations of the KA concentration was done with the DA's calibration curve, under the estimation that the area intensity of the DA's transition $m / z 312.1>266.1$ is approximately 5 times more intense than that of the KA's $m / z 214.1>168.1$.

were analyzed both by the screening LFIAs and the newly developed iMBS-MS/MS method. The results were similar to those of the accredited LC-MS/MS and LC-UV methods for DA. The LFIAs, showed positive results and high contamination levels beyond $1 \times \mathrm{TL}$ according to semi-quantitative smartphone analysis. Also, the iMBS-MS/MS results were in accordance with previous results from the independent reference method. For incurred mussel samples \#1 and \#2, the estimated concentration was close to the analysis result of the reference method. For the incurred mussel \#3, probably because of saturation of the immobilized mAbs on the paramagnetic microspheres, the estimated concentration according to iMBS-MS/MS was somewhat lower but in accordance with the limited dynamic range of an immunocapturing method. In all cases, however, the iMBS-MS/MS results correctly characterized the analyzed samples as noncompliant and containing DA, confirmed by the respective ion ratio for DA $(m / z 248.1 / 266.1)$ within the $0.34 \pm 40 \%$ tolerance limit set by the legislation, ${ }^{15}$ and not containing the harmless KA (Table 1).

\section{CONCLUSIONS}

Ambient and direct ionization mass spectrometric techniques are rapid tools for detecting numerous substances, as many applications demonstrate. However, their inherent lack of chromatographic separation leads to their exclusion as confirmatory methods in EU food-safety schemes, as they do not meet the standards laid down in legislation. In this work, we have demonstrated the development of an iMBS-MS/MS confirmatory analysis method and its validation according to the very recently revised EU legislation. Moreover, we showed that iMBS-MS/MS rapidly identifies false-positive LFIA screening results caused by harmless unregulated structure analogs. The iMBS exploits the use of mAbs for selective isolation of the analyte of interest, adding substantially to the overall specificity of the rapid direct MS/MS approach, thereby competing with time-consuming regulatory LC-MS/MS methods. An additional IP should be granted in future revisions of the legislation, because of the "immunochromatography" nature of the iMBS-MS/MS approach. The developed method is generic, reproducible, and quantitative without employing an I.S. and could be applied to any MSamenable analyte, provided that a pair of antigen/antibody is available. Moreover, different sets of immuno-enriched paramagnetic microspheres with antibodies aiming at different analytes could lead to multiplex iMBS-MS/MS confirmatory analysis opportunities, complementary to multiplex planar array immuno assays used for parallel screening of routine samples.

\section{ASSOCIATED CONTENT}

\section{(s) Supporting Information}

The Supporting Information is available free of charge at https://pubs.acs.org/doi/10.1021/acs.analchem.1c03816.

Method development: Optimization of blade spray and MS conditions, calculation of capacity of the immunoenriched paramagnetic microspheres employed in iMBSMS/MS, comparison between non-coated blades and prototype magnetic blades, smartphone analysis of screening LFIAs for DA in shellfish, initial in-house validation, references, (Figure S1) photographs of the experimental setup of iMBS-MS/MS, (Figure S2) spray solution optimization, (Figure S3) comparison between non-coated blades and prototype magnetic blades, (Figure S4) photographs of the smartphone reading of commercial LFIA, (Figure S5) iMBS-MS/MS chronograms of the $\mathrm{m} / \mathrm{z} 312.1>266.1$., (Figure S6) matrixmatched calibration curves for iMBS-MS/MS measurements, (Table 1) validation results from iMBS-MS/MS conformatory analysis and LFIA screening (PDF)

\section{AUTHOR INFORMATION}

\section{Corresponding Author}

Ariadni Geballa-Koukoula - Wageningen Food Safety Research, Wageningen University and Research, $6700 \mathrm{AE}$ Wageningen, The Netherlands; 0 orcid.org/0000-00031327-6690; Email: ariadni.geballakoukoula@wur.nl

\section{Authors}

Arjen Gerssen - Wageningen Food Safety Research, Wageningen University and Research, $6700 \mathrm{AE}$ Wageningen, The Netherlands

Marco H. Blokland - Wageningen Food Safety Research, Wageningen University and Research, 6700 AE Wageningen, The Netherlands; ๑ orcid.org/0000-0002-3751-6065

Christopher T. Elliott - ASSET Technology Centre, Institute for Global Food Security, School of Biological Sciences, Queen's University Belfast, Belfast BT9 5DL Northern Ireland, U.K.; $\odot$ orcid.org/0000-0003-0495-2909

Janusz Pawliszyn - Department of Chemistry, University of Waterloo, Waterloo, Ontario N2L 3G1, Canada; (1) orcid.org/0000-0002-9975-5811

Michel W.F. Nielen - Wageningen Food Safety Research, Wageningen University and Research, 6700 AE Wageningen, 
The Netherlands; Laboratory of Organic Chemistry, Wageningen University, 6708 WE Wageningen, The Netherlands; (1) orcid.org/0000-0003-4634-0249

Complete contact information is available at: https://pubs.acs.org/10.1021/acs.analchem.1c03816

\section{Author Contributions}

A.G.K. participated in experimental research, data analysis, validation, wrote the original draft, and reviewed and edited the paper. A.G. and M.H.B. supervised and reviewed the paper. C.E. reviewed the paper and provided LFIA expertise. J.P. reviewed the paper and provided CBS expertise. M.W.F.N. contributed in conceptualization, providing resources, reviewing and editing, supervision, and project administration. All authors have given approval to the final version of the manuscript.

\section{Funding}

This project has received funding from the European Union's Horizon 2020 research and innovation program under the Marie Sklodowska-Curie grant agreement no. 720325 and the Dutch Ministry of Agriculture, Nature, and Food Quality under project KB-37-002-005.

\section{Notes}

The authors declare no competing financial interest.

\section{ACKNOWLEDGMENTS}

We would like to thank Liza Portier and Xu Mang from Wageningen Food Safety Research, for assistance with preliminary planar array immunoassay measurements; Sidharam Pujari and Georgina M.S. Ross from Wageningen University for providing the SEM images and the 3D-printed smartphone holder, respectively. Also, the authors wish to thank Professor Katrina Campbell from Queen's University Belfast for supplying the domoic acid monoclonal antibody used in this study.

\section{REFERENCES}

(1) Hird, S. J.; Lau, B. P. Y.; Schuhmacher, R.; Krska, R. TrAC, Trends Anal. Chem. 2014, 59, 59-72.

(2) Greer, B.; Chevallier, O.; Quinn, B.; Botana, L. M.; Elliott, C. T. TrAC, Trends Anal. Chem. 2021, 141, 116284.

(3) Silano, M.; Silano, V. Crit. Rev. Food Sci. Nutr. 2017, 57, 21622217.

(4) Tsagkaris, A. S.; Nelis, J. L. D.; Ross, G. M. S.; Jafari, S.; Guercetti, J.; Kopper, K.; Zhao, Y.; Rafferty, K.; Salvador, J. P.; Migliorelli, D.; Salentijn, G. I.; Campbell, K.; Marco, M. P.; Elliot, C. T.; Nielen, M. W. F.; Pulkrabova, J.; Hajslova, J. TrAC, Trends Anal. Chem. 2019, 121, 115688.

(5) Beneito-Cambra, M.; Gilbert-López, B.; Moreno-González, D.; Bouza, M.; Franzke, J.; García-Reyes, J. F.; Molina-Díaz, A. Anal. Methods 2020, 40, 4831-4852.

(6) So, P.-K.; Hu, B.; Yao, Z.-P. Mass Spectrom. 2014, 3, S0028S0028.

(7) Cody, R. B.; Laramée, J. A.; Durst, H. D. Anal. Chem. 2005, 77, 2297-2302.

(8) Takáts, Z.; Wiseman, J. M.; Gologan, B.; Graham Cooks, R. Science 2004, 306, 471-473.

(9) Gómez-Ríos, G. A.; Pawliszyn, J. Angew. Chem. 2014, 53, $14503-14507$.

(10) Wang, H.; Liu, J.; Graham Cooks, R.; Ouyang, Z. Angew. Chem. 2010, 49, 877-880.

(11) Hu, B.; So, P. K.; Yang, Y.; Deng, J.; Choi, Y. C.; Luan, T.; Yao, Z. P. Anal. Chem. 2018, 90, 1759-1766.
(12) Blokland, M. H.; Gerssen, A.; Zoontjes, P. W.; Pawliszyn, J.; Nielen, M. W. F. Food Anal. Methods 2020, 13, 706-717.

(13) Jager, J.; Gerssen, A.; Pawliszyn, J.; Sterk, S. S.; Nielen, M. W. F.; Blokland, M. H. J. Am. Soc. Mass Spectrom. 2020, 31, 2243-2249.

(14) Rickert, D. A.; Singh, V.; Thirukumaran, M.; Grandy, J. J.; Belinato, J. R.; Lashgari, M.; Pawliszyn, J. Environ. Sci. Technol. 2020, 54, 15789-15799.

(15) European Commission. Off. J. Eur. Union. 2021, 180, 84-109.

(16) Jafari, S.; Guercetti, J.; Geballa-Koukoula, A.; Tsagkaris, A. S.; Nelis, J. L. D.; Marco, M.-P.; Salvador, J.-P.; Gerssen, A.; Hajslova, J.; Elliott, C.; Campbell, K.; Migliorelli, D.; Burr, L.; Generelli, S.; Nielen, M. W. F.; Sturla, S. J. Foods 2021, 10, 1399.

(17) Pérez-López, B.; Mir, M. Talanta 2021, 225, 121898.

(18) Xiao, X.; Hu, S.; Lai, X.; Peng, J.; Lai, W. Trends Food Sci. Technol. 2021, 111, 68-88.

(19) Fraga, M.; Vilarińo, N.; Louzao, M. C.; Rodríguez, P.; Campbell, K.; Elliott, C. T.; Botana, L. M. Anal. Chem. 2013, 85, 7794-7802.

(20) Graham, H.; Chandler, D. J.; Dunbar, S. A. Methods 2019, 158, $2-11$.

(21) Qu, J.; Xie, H.; Zhang, S.; Luo, P.; Guo, P.; Chen, X.; Ke, Y.; Zhuang, J.; Zhou, F.; Jiang, W. Food Anal. Methods 2019, 12, 877886.

(22) Zhang, Y.; Li, X.; Nie, H.; Yang, L.; Li, Z.; Bai, Y.; Niu, L.; Song, D.; Liu, H. Anal. Chem. 2015, 87, 6505-6509.

(23) Joshi, S.; Zuilhof, H.; Van Beek, T. A.; Nielen, M. W. F. Anal. Chem. 2017, 89, 1427-1432.

(24) Zhao, Y.; Tang, M.; Liu, F.; Li, H.; Wang, H.; Xu, D. Anal. Chem. 2019, 91, 13418-13426.

(25) Geballa-Koukoula, A.; Gerssen, A.; Nielen, M. W. F. Anal. Bioanal. Chem. 2020, 412, 7547-7558.

(26) Geballa-Koukoula, A.; Gerssen, A.; Nielen, M. W. F. Sensors 2021, 21, 1861.

(27) Lefebvre, K. A.; Robertson, A. Toxicon 2010, 56, 218-230.

(28) Schrenk, D.; Bignami, M.; Bodin, L.; del Mazo, J.; GraslKraupp, B.; Hogstrand, C.; Chipman, K. J.; Leblanc, J.; Nebbia, C. S.; Nielsen, E.; Ntzani, E.; Petersen, A.; Sand, S.; Schwerdtle, T.; Vleminckx, C.; Wallace, H.; Martinez, A. G.; Gerssen, A.; Tubaro, A.; Cascio, C.; Abrahantes, J. C.; Steinkellner, H.; Hoogenboom, L. EFSA J. 2021, 19, 1-57.

(29) European Commission. Off. J. Eur. Union. 2004, 139, 1-14.

(30) Yakes, B. J.; Buijs, J.; Elliott, C. T.; Campbell, K. Talanta 2016, 156-157, 55-63.

(31) Dunbar, S. A.; Hoffmeyer, M. R. Microsphere-Based Multiplex Immunoassays: Development and Applications Using Luminex® XMAP® Technology. The Immunoassay Handbook; Elsevier Ltd, 2013, 157-174.

(32) Jawaid, W.; Meneely, J.; Campbell, K.; Hooper, M.; Melville, K.; Holmes, S.; Rice, J.; Elliott, C. Talanta 2013, 116, 663-669.

(33) Berendsen, B. J. A.; Stolker, L. A. M.; Nielen, M. W. F. J. Am. Soc. Mass Spectrom. 2013, 24, 154-163. 